\title{
SPECTRA OF THE CENTRAL STARS OF PLANETARY NEBULAE
}

\author{
Lawrence H. Aller \\ (University of California, Los Angeles, Calif., U.S.A.)
}

\section{Introduction}

The central stars of planetary nebulae represent seemingly well-defined late stages of stellar evolution. Theoretical investigations with predictions of evolutionary tracks impose difficult requirements for observational data. Measurements of spectral energy distributions, of colors, and of magnitudes, and spectroscopic observations are all urgently needed.

Here one is confronted not only with the usual difficulties attending observations of faint stars, but also with interference from the radiation of the nebulae themselves. The nebular hydrogen and helium lines usually mask the corresponding absorption features of the stellar spectrum. If the nebular spectrum is of low excitation, stellar He II lines such as $\lambda 4686$ or $\lambda 4541$ may sometimes be observed in absorption. Incipient emission in $\lambda 4686$ often fills in the corresponding stellar absorption line. Only for a few stars, such as the nucleus of NGC 246, NGC 1514, and NGC 3132 and the Abell objects (1966) observed by Greenstein and Minkowski (1964) are the nebulae so faint that interference from the nebulae spectrum is not significant.

Observations of the broad-emission-line spectra of central stars of the Wolf-Rayet type are less affected by confusion with nebular lines unless the star is very faint. Many of these stars show lines near $\lambda 4640$ which coincide with radiations of nebular origin, the confusion being particularly serious for stellar planetaries.

Table 1 lists those nebulae whose nuclear spectra have been observed at Lick Observatory. A few are sufficiently bright to be photographed with the coudé spectrograph at the 120-inch. Most of them require observations with the air-Schmidt camera and nebular spectograph at the prime focus. The dispersion at the coudé is $16 \AA / \mathrm{mm}$; at the prime focus the dispersion is $97 \AA / \mathrm{mm}$. Table 2 lists those nebulae whose spectra display a strong continuum and in which no central stars have been observed. It is impossible to tell whether the continuum in some 'stellar' planetaries originates from the nebula or from a central star, but it is certain that in objects such as NGC 2440 or NGC 7027 the continuous spectrum is of nebular origin.

\section{Planetary Nebulae with Continuous and Absorption-Line Spectra}

Many objects listed as exhibiting purely continuous spectra may have weak ab- 
Table 1

\section{Planetary Nebulae Whose Central Stars have been Observed}

\begin{tabular}{|c|c|c|c|c|c|}
\hline Nebula & Description & Notes & Nebula & Description & Notes \\
\hline NGC 40 & WR & $1,2,6$ & NGC 6543 & Of & $1,2,6$ \\
\hline NGC 246 & Of & 1,6 & NGC 6572 & Of? & 1,5 \\
\hline NGC 650 & contin & 4 & NGC 6567 & contin & \\
\hline IC 1747 & WR & 2 & NGC 6629 & $\mathrm{O}, \mathrm{abs}$ & 2 \\
\hline IC 351 & contin & & NGC 6720 & contin & \\
\hline NGC 1501 & WR & 2 & NGC 6751 & WR & 1,2 \\
\hline IC 289 & contin & & NGC 6778 & contin & \\
\hline NGC 1514 & A & $\begin{array}{r}\text { 1, strong } \\
\text { K line }\end{array}$ & $\begin{array}{l}\text { NGC } 6790 \\
\text { NGC } 6803\end{array}$ & $\begin{array}{l}\text { contin } \\
\text { contin }\end{array}$ & 5 \\
\hline NGC 1535 & $\mathrm{O}, \mathrm{abs}$ & 6 & NGC 6804 & $\mathrm{O}, \mathrm{abs}$ & 3 \\
\hline J 320 & & 5 & $\mathrm{BD}+303639$ & WR & $1,2,6$ \\
\hline IC 418 & Of & 1,2 & NGC 6818 & contin & \\
\hline NGC 2022 & contin & & NGC 6826 & Of & $1,2,6$ \\
\hline IC 2149 & $\mathrm{O}, \mathrm{abs}$ & 1 & NGC 6853 & $\mathrm{O}, \mathrm{abs}$ & 3 \\
\hline NGC 2371-2 & WR & & NGC 6884 & contin? & 5 \\
\hline NGC 2346 & A & & NGC 6886 & contin & 4 \\
\hline NGC 2392 & Of & $1,2,6$ & NGC 6891 & Of & 1,2 \\
\hline NGC 2438 & contin & & IC 4997 & Of & $1,5,6$ \\
\hline NGC 2452 & WR & 2,4 & NGC 6905 & WR & 1,2 \\
\hline NGC 3132 & A & strong K & NGC 7008 & $\mathrm{O}$, abs & 3 \\
\hline NGC 3242 & contin & 6 & NGC 7009 & contin & 6 \\
\hline NGC 4361 & $\mathrm{O}, \mathrm{abs}$ & 3 & NGC 7026 & WR & 2 \\
\hline IC 3568 & $O, a b s$ & 3 & IC 5217 & contin? & 5 \\
\hline NGC 6058 & $\mathrm{O}, \mathrm{abs}$ & 3 & NGC 7354 & contin & 4 \\
\hline IC 4593 & Of & 1,6 & NGC 7662 & contin & 6 \\
\hline NGC 6210 & Of & 1,6 & $C D-32^{\circ} 14673$ & $\mathrm{O}$, abs & \\
\hline IC 4634 & contin? & & VV 286 & contin? & 5 \\
\hline NGC 6309 & contin & & & & \\
\hline NGC 6445 & contin & 4 & & & \\
\hline
\end{tabular}

WR $=$ Wolf-Rayet type.

contin $=$ continuous with no absorption or emission.

$\mathrm{O}, \mathrm{abs}=$ continuous with absorption lines.

Of $=$ continuous with narrow emission lines and possibly also absorption features.

Notes:

1 Photometric measurements and/or detailed descriptions have been published (Aller, 1948, 1956; Andrillat, 1959; Kohoutek, 1966; Swings, 1940; Swings and Struve, 1940; Swings and Swensson, 1952; Wilson, 1948; Wilson and Aller, 1954).

2 Photometric measurements and descriptions are in preparation.

3 Absorption-line intensities are listed in Table 3.

${ }^{4}$ Spectrum is very weak.

5 Stellar origin not certain or nebular continuous spectrum may seriously influence stellar spectrum.

${ }^{6}$ Observed with coudé dispersion, $16 \AA / \mathrm{mm}$. 


\section{Table 2}

Nebulae with Strong Continuous Spectra for which no Central Stars have been Observed

Anon $0^{\mathrm{h}} 26^{\mathrm{m}}$
IC 2165
NGC 2440
J 900

NGC 6537
NGC $6644^{1}$
IC $4732^{1}$
IC $4776^{1}$

NGC 6537

IC $4776^{1}$

\author{
NGC 6741 \\ NGC $6833^{1}$ \\ NGC 7027 \\ IC $5117^{1}$
}

${ }^{1}$ Stellar origin not certain or nebular continuous spectrum may seriously influence stellar spectrum.

sorption lines which, at the dispersions employed, are masked by the overlying nebular emission. Emission lines, characteristic of Of stars may also be missed if they coincide with strong nebular lines. We can assert with some confidence that bright stars such as the nuclei of NGC 3242, NGC 7009, and NGC 7662 have purely continuous spectra with no detectable trace of emission or absorption features.

Observations of the spectra of the brighter absorption-line stars, such as the nucleus of NGC 6891, 6826, IC 4593, and NGC 6210 substantiate earlier studies (Aller, 1948; Wilson and Aller, 1954) and are being analysed now by Miss Sarah Heap. Table 3

Table 3

\section{Absorption-Line Spectra of Several Planetary Nuclei Intensities expressed in equivalent widths $W_{\lambda}$}

$\lambda \quad$ NGC $4361 \quad$ II $3568 \quad$ NGC $6058 \quad$ NGC $6804 \quad$ NGC $6853 \quad$ NGC 7008

\begin{tabular}{|c|c|c|c|c|c|c|}
\hline 4861 & & $2 \cdot 1$ & & & & $1 \cdot 8$ \\
\hline 4686 & 1.5 & & & 3. & 2.0 & 1.4 \\
\hline 4541 & 0.9 & 0.8 & & & & \\
\hline 4340 & 1.7 & 1.0 & $2 \cdot 7$ & $2 \cdot 7$ & 1.7 & 1.8 \\
\hline 4200 & & 0.5 & & & & \\
\hline $4101 \cdot 7$ & $1 \cdot 6$ & 0.6 & $2 \cdot 6$ & $2 \cdot 7$ & 0.7 & $2 \cdot 1$ \\
\hline 3970 & $1 \cdot 2$ & 0.8 & $2 \cdot 0$ & 1.2 & & 1.7 \\
\hline 3933 & & & & & & 0.4 \\
\hline 3889 & $1 \cdot 1$ & 1.0 & 1.7 & 1.0 & 0.9 & $1 \cdot 1$ \\
\hline 3835 & 1.0 & 0.6 & 1.5 & & & 0.8 \\
\hline 4797 & & 0.4 & 1.2 & & & 0.7 \\
\hline 3770 & & 0.2 & 1.0 & & & $0 \cdot 6$ \\
\hline 3750 & & & 0.3 & & & 0.4 \\
\hline $\begin{array}{l}\text { Suggested } \\
\text { Spectral } \\
\text { Class }\end{array}$ & 06 & 05 & $09 ?$ & 09 & 07 & 07 \\
\hline
\end{tabular}

lists approximate intensities expressed as equivalent widths for stars observed at the prime focus. At a dispersion of $97 \AA / \mathrm{mm}$ with an overlapping nebular line spectrum, only rough estimates are possible. Furthermore, note that the presence of an overlying 
nebular continuum causes all of the tabulated equivalent widths to be lower limits. Therefore, although the tabulated intensities may be useful for estimating spectral classes, they should be regarded as little better than eye estimates. Generally, I widened the spectra by drifting, but only a small degree of widening is possible for the spectrum of NGC 4361 and the tabulated intensities are, therefore, particularly uncertain.

In $C D-32^{\circ} 14673\left(\alpha=18^{\mathrm{h}} 53^{\mathrm{m}} 24^{\mathrm{s}}, \delta=-32^{\circ} 17^{\prime}\right)$, the nebular Balmer lines completely mask any stellar hydrogen features, while the Pickering $\lambda 4541$ and $\lambda 4200 \mathrm{He}$ II lines have equivalent widths of $0.6 \AA$ and $0.5 \AA$, respectively.

Further studies of these objects should be carried out with high dispersion and an image converter. Walker and Aller have carried out a few preliminary tests at the coudé focus of the 120-inch.

\section{The Spectrum of the Nucleus of NGC 6543}

Descriptions of the spectrum of the central star of NGC 6543 have been given by Swings (1940); for quantitative estimates of line intensities see Aller (1943, 1956, p. 211). With the coudé spectrograph of the 120 -inch it is possible to measure not only the total intensities of the nebular lines but also their profiles.

With the higher dispersion and decreased contamination from the nebular spectrum gained at the coudé focus, $\mathrm{H} \gamma$ and $\mathrm{H} \delta$ show profiles suggestive of the P Cygni type. The higher members of the series appear in absorption. The Balmer-line profiles are complicated because they are blended with the lines of ionized helium. Hence the centers appear displaced from the nebular emission lines.

Table 4 compares the spectrum observed at three different dates: In 1945 with a Cassegrain spectrograph at McDonald Observatory (Aller, 1956) and on June 18 and August 16, 1965, at Lick Observatory. In 1945 the NV $\lambda 4603$ and $\lambda 4619$ lines seemed to show P Cygni characteristics; later they seemed to be mostly in emission. Some change was noted in the appearance of complex structures between $\lambda 4630$ and $\lambda 4659$. The OIII, OIV, NiII, and Niv lines observed shortward of $\lambda 3760$ show comparable intensities, although there is some hint that the $3 \mathrm{~s}^{3} \mathrm{~S}-3 \mathrm{p}^{3} \mathrm{P}$ transitions were stronger on plate ES 4325 than on either other date of observation. The $\lambda 4686 \mathrm{He}$ II line shows definite intensity and profile variations.

Line intensity and profile changes are well known in classical Wolf-Rayet stars where they may be of very short duration, Oke (1954). Probably the variations in planetary nebulae nuclei are also of very short duration (Wilson and Aller, 1954), but we have no easy means of proving this - unless an image converter can be used.

Thus as has been suggested often, it seems likely that the Of nuclei are stars with unstable atmospheres which are ejecting material into the surrounding nebular shells at the present time. 


\section{Table 4}

\section{The Spectrum of the Nucleus of NGC 6543}

\begin{tabular}{|c|c|c|c|c|}
\hline$\lambda$ & Ion & 1945 & $\begin{array}{c}\text { June } 1965 \\
\text { EC } 4329\end{array}$ & $\begin{array}{r}\text { Aug } 1965 \\
\text { EC } 4458\end{array}$ \\
\hline $4685 \cdot 8$ & HeII & $13 \cdot 8$ & $9 \cdot 1$ & $12 \cdot 6$ \\
\hline $4658 \cdot 6$ & CIV & 2.5 & 0.6 & 1.6 \\
\hline 4648 & CIII & $1 \cdot 6$ & $\mathrm{pr}$ & $0 \cdot 6$ : \\
\hline 4641 & NiII & & 0.74 & 0.74 \\
\hline \multirow[t]{2}{*}{4632} & $N_{\text {III }}$ & & 0.5 & 0.6 \\
\hline & & 0.4 & 0.35 & 0.3 ? \\
\hline \multirow{2}{*}{4619.4} & $\mathrm{Nv}$ & $0 \cdot 2 \mathrm{a}$ & $\mathrm{pr}$ & $\mathrm{pr}$ \\
\hline & & 0.7 & 0.99 & 0.79 \\
\hline $4603 \cdot 2$ & $\mathrm{~N} v$ & $0 \cdot 3 a$ & - & $\mathrm{pr}$ \\
\hline \multirow{2}{*}{$4541 \cdot 6$} & HeII & $0 \cdot 8 \mathrm{a}$ & & \\
\hline & & & $0 \cdot 3:$ & $0 \cdot 3$ : \\
\hline 4340 & $\mathbf{H} \gamma$ & & $0.4 \mathrm{a}:$ & $0 \cdot 4 a:$ \\
\hline 4116 & Silv & & $0 \cdot 20$ & 0.2 \\
\hline \multirow[t]{2}{*}{4101} & $\mathrm{H} \delta$ & & $0 \cdot 3:$ & $0 \cdot 3:$ \\
\hline & & & $0.5 \mathrm{a}:$ & $0 \cdot 5 \mathrm{a}:$ \\
\hline \multirow{2}{*}{4088} & SiIV & 0.8 & 0.6 & 0.5 \\
\hline & & 1.0 & & \\
\hline 4058 & Niv & $0 \cdot 16 \mathrm{a}$ & $1 \cdot 2$ & 1.6 \\
\hline 3970 & $\mathrm{H}, \mathrm{He}$ & & $1 \cdot 24 \mathrm{a}$ & $1.6 \mathrm{a}$ \\
\hline 3889 & $\mathrm{H}, \mathrm{He}$ & $1.3 \mathrm{a}$ & $1 \cdot 1 \mathrm{a}$ & $0.7 \mathrm{a}$ \\
\hline 3835 & $\mathrm{H}$ & $0.6 \mathrm{a}$ & $0.7 \mathrm{a}$ & $0.7 \mathrm{a}$ \\
\hline 3797 & $\mathrm{H}$ & $0.7 \mathrm{a}$ & $0.6 \mathrm{a}$ & $0.7 \mathrm{a}$ \\
\hline 3771 & & & 0.5 & $0.5 \mathrm{a}$ \\
\hline 3760 & OIII & & $0 \cdot 3$ & $0 \cdot 3$ \\
\hline 3757 & OIII & & $0 \cdot 2$ & 0.4 \\
\hline 3755 & $\mathrm{O}_{\text {III }} / \mathrm{N}_{\text {III }}$ & & 0.3 & 0.4 \\
\hline 3737 & OIV & & 0.3 & 0.4 \\
\hline 3714 & NiV & & 0.4 & 0.4 \\
\hline 3563.4 & OIV & 0.6 & $0 \cdot 6$ & 0.6 \\
\hline $3560 \cdot 9$ & OIV & 0.7 & $0 \cdot 6$ & $0 \cdot 8$ \\
\hline 3555 & HeI & - & 0.3 & 0.2 \\
\hline 3484.9 & Niv & 0.4 & 0.9 & 0.5 \\
\hline 3483.0 & Niv & 0.5 & $0 \cdot 8$ & 0.5 \\
\hline $3478 \cdot 7$ & Niv & 0.5 & 1.6: & 0.6 \\
\hline $3409 \pm 3411 \cdot 8$ & OIV & $2 \cdot 1$ & $2 \cdot 8$ & $2 \cdot 4$ \\
\hline $3403 \cdot 6$ & OIV & 0.8 & 1.0 & 1.0 \\
\hline $3396 \cdot 8$ & OIV & 0.2 & 0.6 & 0.5 \\
\hline $3385 \cdot 5$ & OIV & $0 \cdot 3$ & 0.7 & $0 \cdot 4$ \\
\hline $3381 \cdot 3$ & OIV & 0.3 & & \\
\hline 3375 & OIV & 0.4 & $0 \cdot 3$ : & \\
\hline
\end{tabular}

An absorption feature is denoted by ' $a$ '. 


\section{Spectra of the Wolf-Rayet Type}

We summarize here only some general features of the spectra of the Wolf-Rayet-like nuclei. Since the pioneer studies of the nuclei of NGC 40 (Paddock, 1915) and of BD
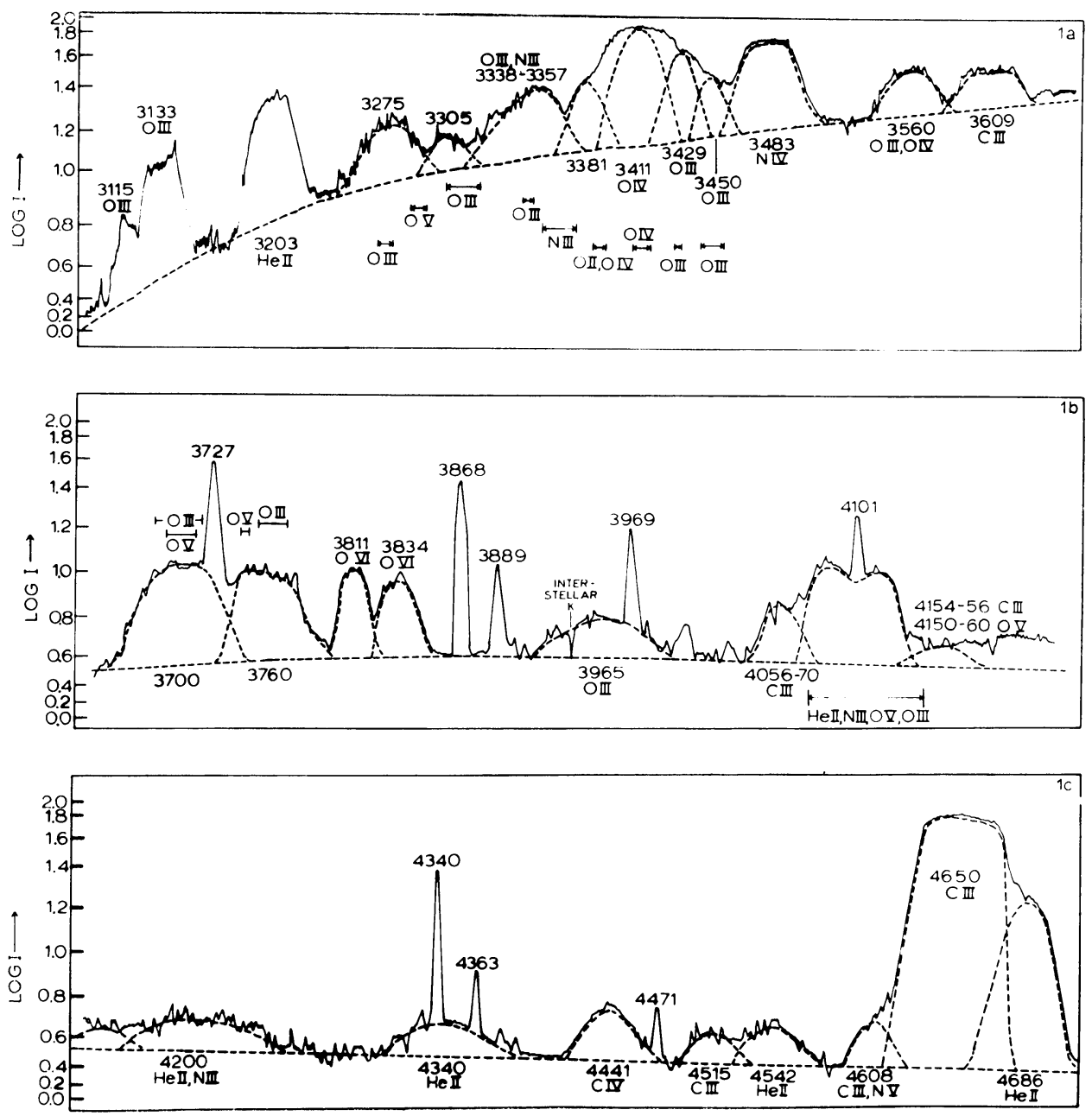

Fig. 1. Tracings of the spectra of the Nuclei of NGC $6751, \lambda 3100-\lambda 4400$. In this and following figures the position of the continuum and suggested resolution of some complex emission structures are indicated by dotted lines. Lines of nebular origin appear as sharp spikes. A logarithmic scale of intensities is indicated at the left.

FiG. 2. Comparison of the $\lambda 4400-\lambda 4700$ region in the central stars of NGC 6905, NGC 7026, IC 1747, IC 2003 and NGC 2371-72. Note the great strength of the emission features in NGC 6905, NGC 7026 and IC 1747. In IC 2003, the emission features of the nuclear spectrum are overwhelmed by the strong lines in the nebular spectrum. 

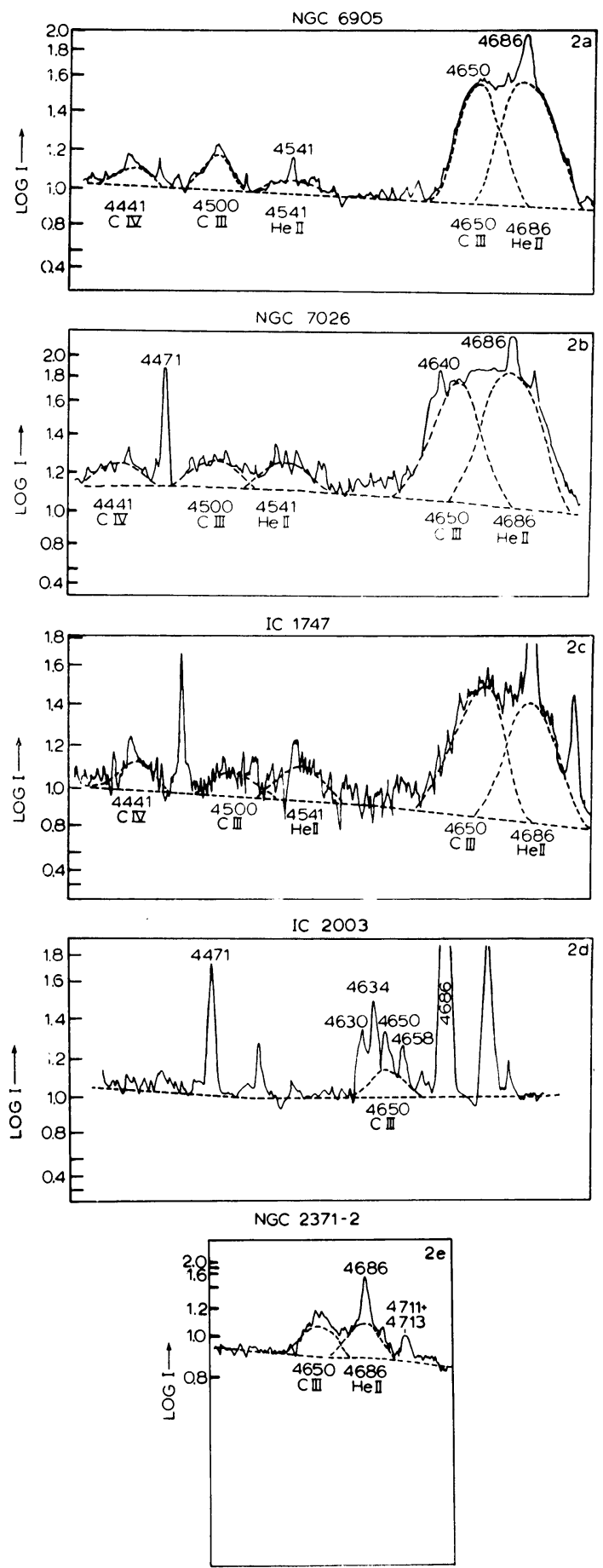

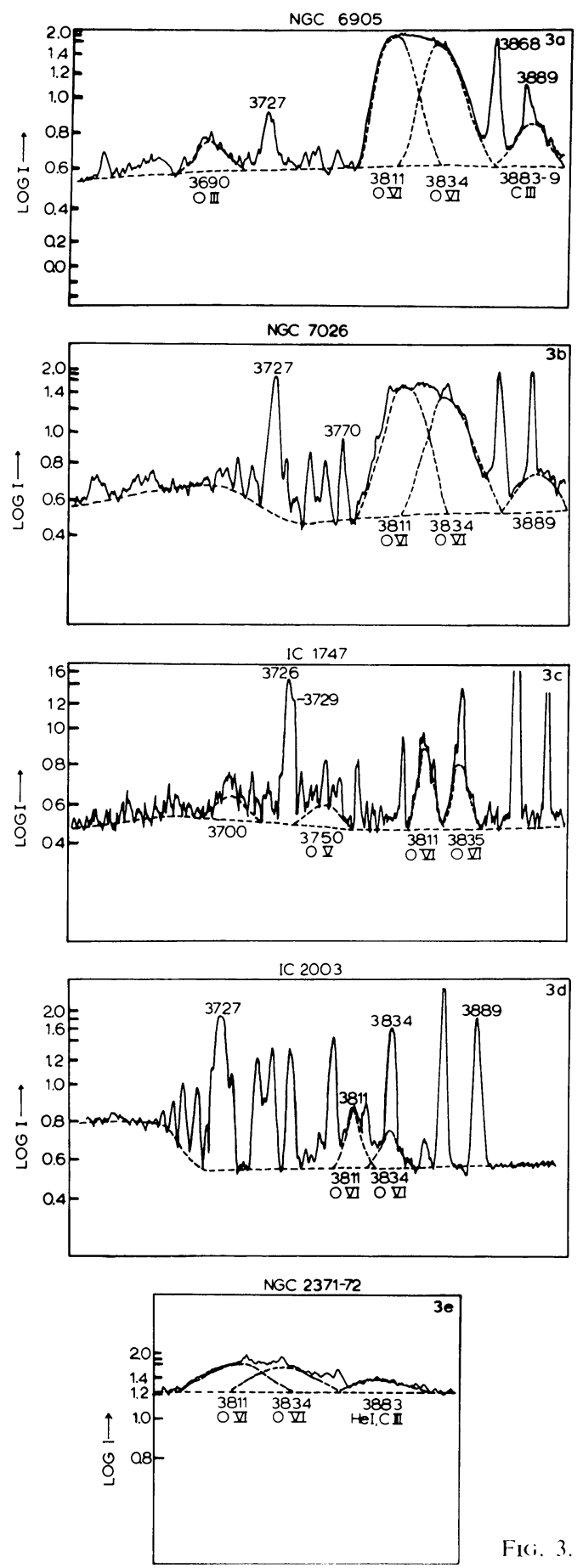

FIci. 3. 
$+30^{\circ} 3639$ (Campbell, 1894) many investigations of these objects have been published (for a summary of earlier work see e.g. Aller, 1956, p. 205-209).

A full discussion of these Wolf-Rayet spectra must await the completion of a study now being conducted jointly by the author and Miss Lindsey Smith at Lick Observatory. In this program we compare the spectra of the planetary nuclei with those of classical Wolf-Rayet stars. Some general remarks can be supplied at this time.

Figure 7 compares the spectra of several planetary nuclei of the Wolf-Rayet type as photographed with the Lick prime focus nebular spectrograph. The spectrum of a B9 star, $\theta$ Crateris, is given for comparison. In Figures 1-4 we reproduce tracings of
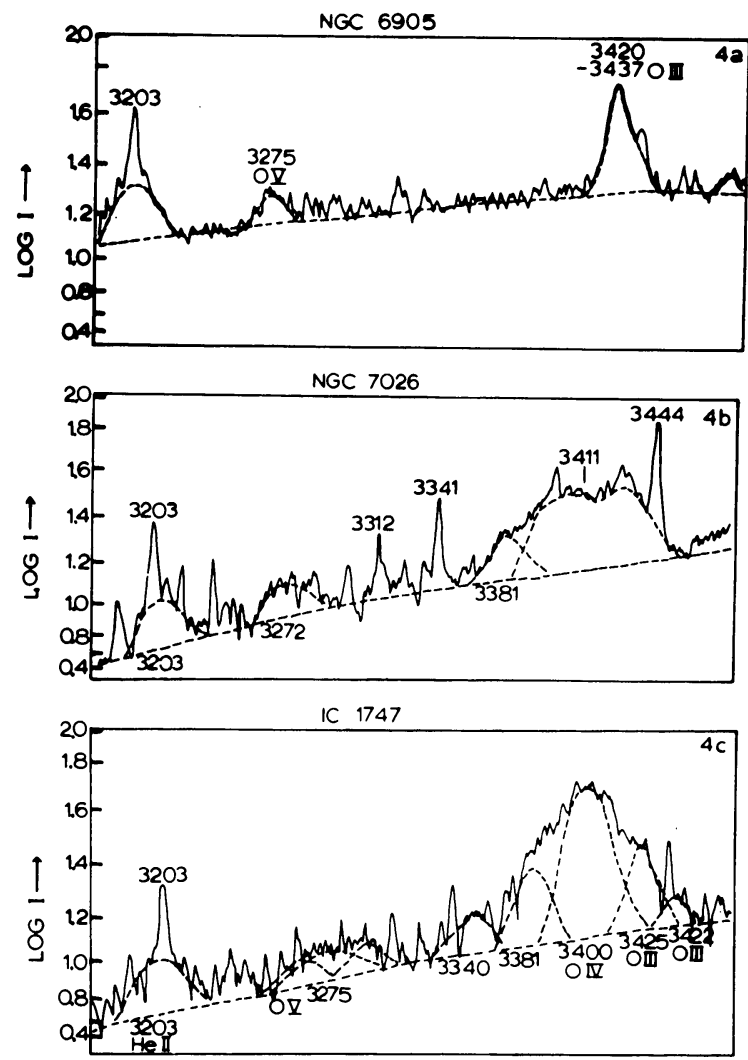

FIG. 4. The ultraviolet region of the spectra of the central stars of NGC 6905, NGC 7026 and IC 1747. The $O_{111}$ lines ( $\left.\lambda 3420-3437\right)$ in NGC 6905 are narrow compared with the complex structures in NGC 7026 and IC 1747. The dotted lines indicate a possible resolution of this structure into separate components.

FIG. 3. The OVI $\lambda 3811,3834$ features in the central stars of NGC 6905, NGC 7026, IC 1747, IC 2003, and NGC 2371-72. The broad OVI lines attain great strength in the nuclei of NGC 6905 and NGC 7026, but are weaker in IC 1747, and in IC 2003 where the continuum is largely of nebular origin. In the central star of NGC 2371-72, the $O \mathrm{v}_{\mathrm{I}}$ lines have broad profiles upon which are superposed narrow emission lines. 

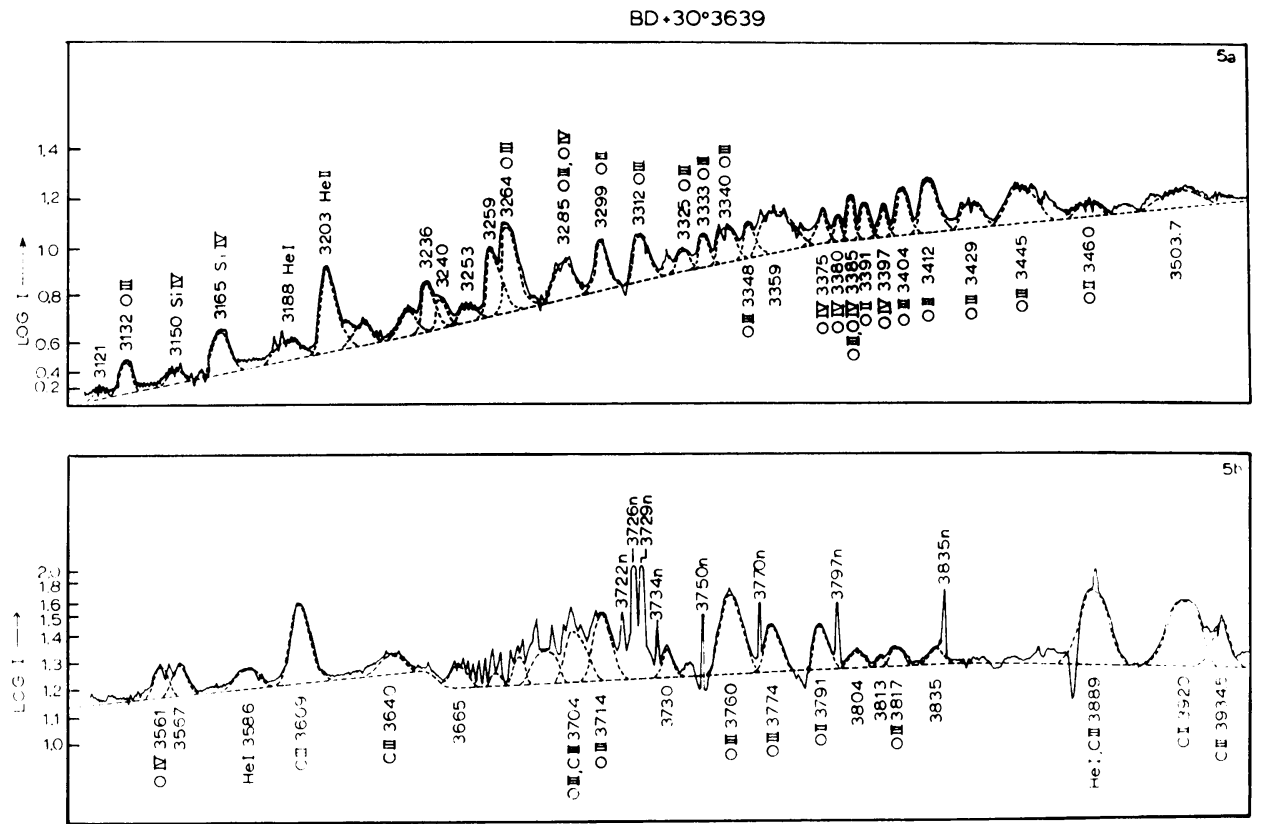

$B D+30^{\circ} 3639$
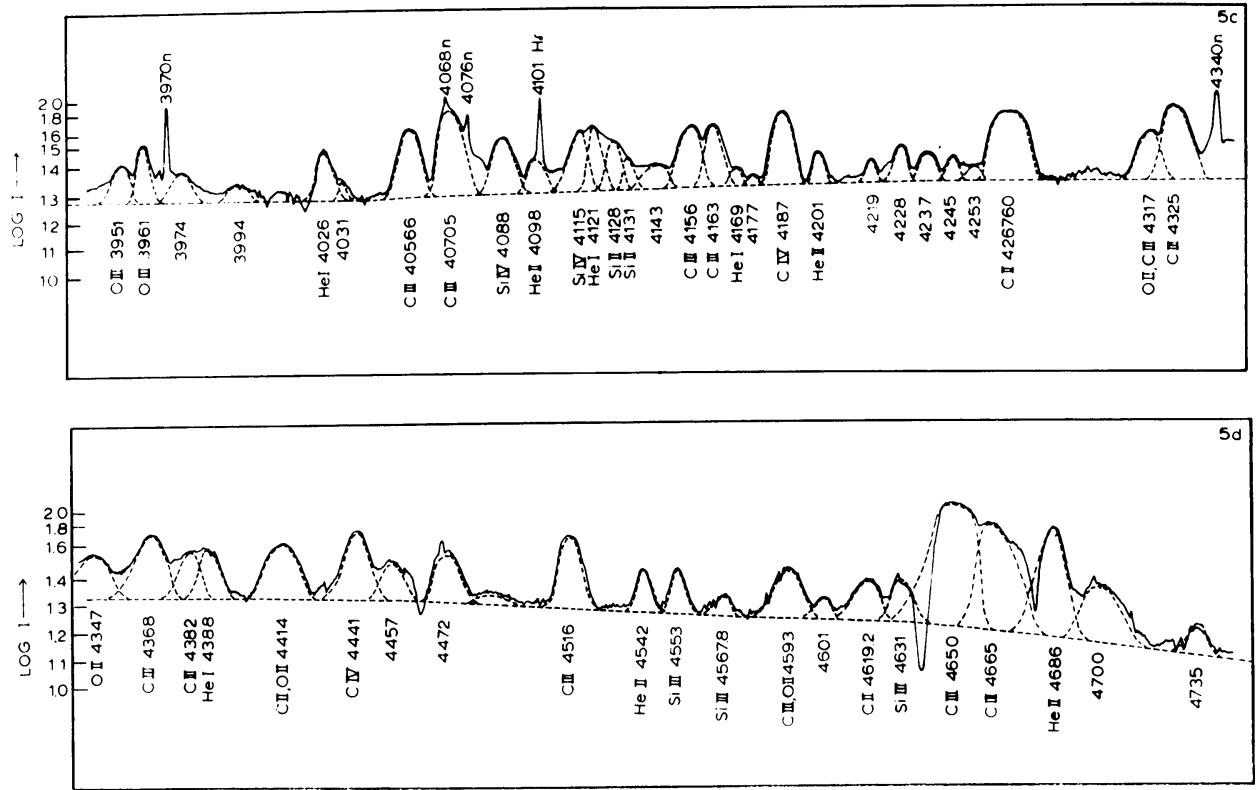

FIG. 5a. 
$B D+30^{\circ} 3639$
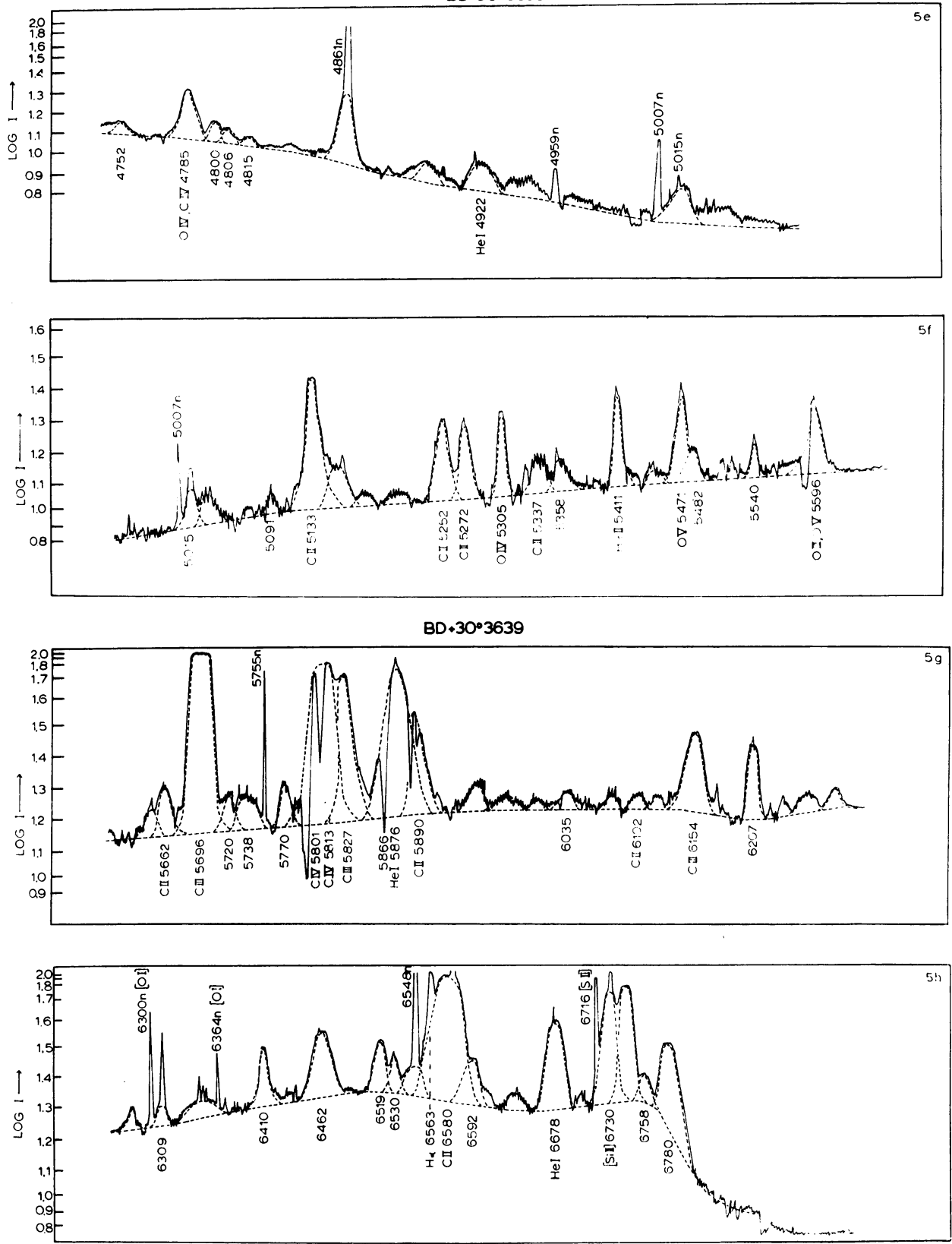

FIG. 5b.

FIG. 5. A tracing of the spectrum of the central star of $B D+30^{\circ} 3639, \lambda 3100-\lambda 6800$. The spectrum is characterized by the sharpness of its lines. Note the absorption features on the violet edges of the lines of $\mathrm{He} \lambda 3889, \mathrm{C} \mathrm{III} \lambda 4650, \mathrm{CIV} \lambda 5801$, and HeI $\lambda$ 5876. Tracings a-e were made from plates secured with an Eastman IIaO emulsion; $f, g$, and $h$ on plates secured with an Eastman IIaF emulsion. 
the more interesting regions of the spectra. Figure 1 shows a tracing of the spectrum of the nucleus of NGC 6751; this strongly resembles spectra of classical Wolf-Rayet stars of the carbon sequence, although N IV $\lambda 3483$ is present and strong. The dotted lines indicate the estimated position of the continuum and the suggested resolution of several strong lines into their respective components. The (relative) $\log _{\text {I }}$ scale is indicated on the left, but no attempt has been made to allow for the wavelength variations of the transmission of the atmosphere and the spectrograph, or for the wavelength variation of the system sensitivity.

Figures $2 \mathrm{a}-\mathrm{e}$ compare the $\lambda 4430-\lambda 4700$ regions of the spectra of the nuclei of NGC 6905, NGC 7026, IC 1747, IC 2003, and NGC 2371-2. In Figures 3a-e a similar comparison is made of the $\lambda 3650-\lambda 3900$ regions, while Figures $4 \mathrm{a}-\mathrm{c}$ compare the ultraviolet regions of the spectra of the nuclei of NGC 6905, NGC 7026, and IC 1747. The spectra have been arranged in order of decreasing strength of the OVI doublet $\lambda 3811, \lambda 3834$. In the spectrum of the nucleus of NGC 2371-2 these lines display sharp cores and broad wings. The spectrum of the nucleus of NGC 246 represents a further step in this sequence; in this spectrum the Ovi lines are very sharp and weak with no underlying broad feature.

Quantitative comparison is rendered difficult by the fact that the continua are strongly affected by contributions from the nebulae as well as by the presence of overlying emission lines. The great strength and dominance of the $\mathrm{O} v \mathrm{r}$ lines in some of these spectra is not paralleled among the spectra of the classical Wolf-Rayet stars.

The nuclei of NGC 1501 and NGC 2452 are also objects of this peculiar Wolf-Rayet type, resembling the nucleus of NGC 6905, with spectra displaying strong broad OVI lines and broad C III $\lambda 4650$ as the dominant features.

Campbell's hydrogen envelope star is, however, characterized by relatively narrow emission lines. Figure 5 displays tracings of the spectrum from $\lambda 3121$ to $\lambda 6800$. The presumed background continuum is indicated by the dotted line; it seems to be fairly well defined, except in the red where the plate sensitivity changes rapidly with wavelength. Notice the absorption lines associated with metastable levels of HeI, $\lambda 3889$, C III $\lambda 4650, C_{\text {IV }} \lambda 5801,5813$, and also $\lambda 5876$. The carbon lines, especially C II $\lambda 5696$ and 6580 and $\mathrm{C}_{\text {III }} \lambda 4650$, dominate the spectrum. A detailed comparison of this stellar spectrum with that of its nearest classical WR analogue is now in progress.

The nucleus of NGC 40 is also a Wolf-Rayet star of the carbon sequence, with numerous broad, strong lines of helium, carbon, and oxygen ions. Except for the wavelength-dependent effects of plate sensitivity, transmission of optics, and atmospheric transmission, the tracings are on a true intensity scale. For the region $\lambda 4650$ to $\lambda 5900 \mathrm{I}$ am indebted to Merle Walker for observations with the Lallemand electronic camera; in the green spectral region the star is too faint to be recorded at the coudé on the usual emulsions in reasonable exposure times.

Czyzak and Aller have obtained photoelectric scans of the spectra of both BD $+30^{\circ}$ 3639 and NGC 40 with the Cassegrain spectrum scanner at the 60-inch telescope on 

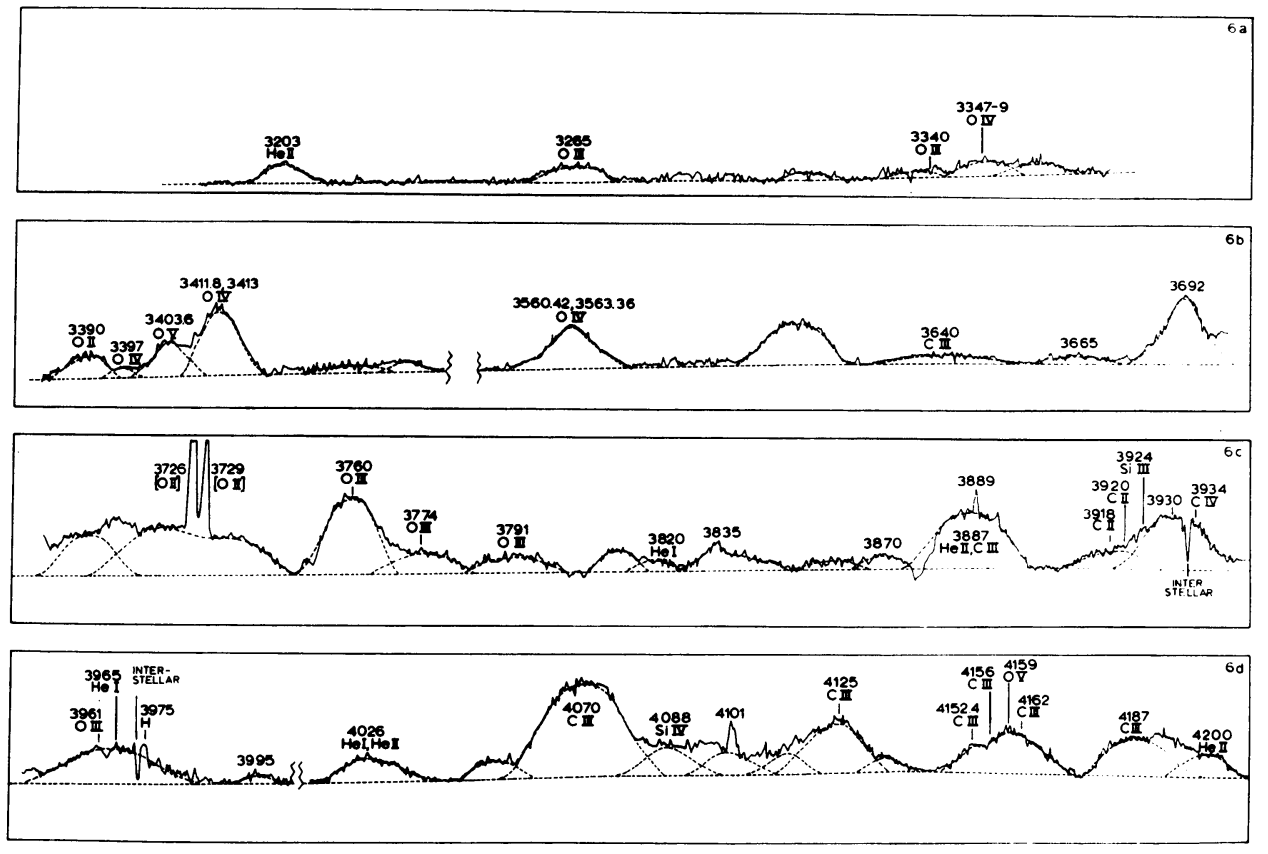

NGC 40 NUCLEUS
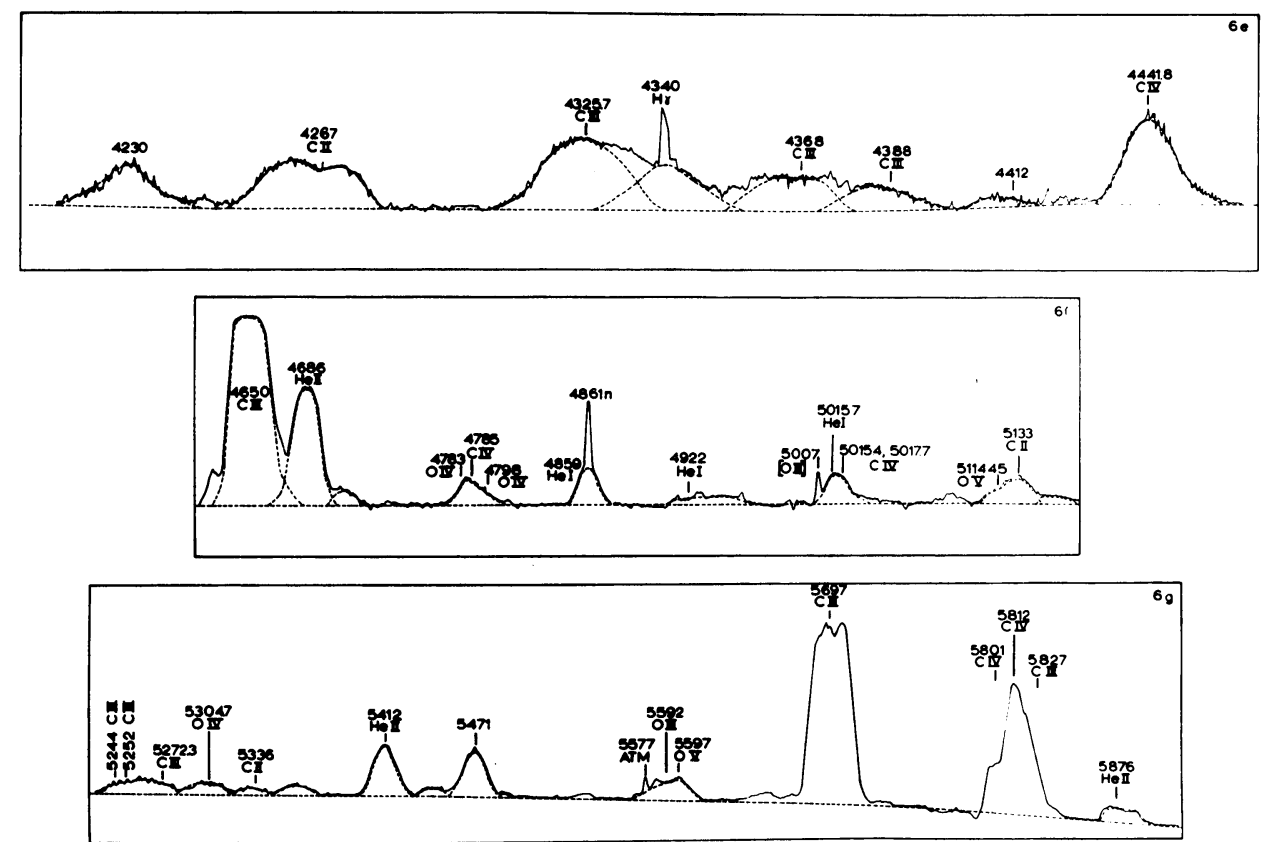

FIG. 6. Tracing of the nuclear spectrum of NGC 40. The tracings are on a direct intensity scale. The region $\lambda 4650-\lambda 5876$ was observed with the Lallemand cell in collaboration with Merle F. Walker. 
Mt. Wilson. These data will make it possible to put all the photographic relative intensity measurements on an absolute scale.

\section{Comments on the Hertzsprung Russell Diagram}

A number of workers have prepared $\log L / L_{\odot}$ vs. $\log T_{\text {eff }}$ plots for the central stars of planetary nebulae. Uncertainties in the bolometric corrections make such plots highly subjective. The well-known discordances between the temperatures appropriate to the spectra of absorption line stars and the 'Zanstra temperatures' of these same objects as obtained by Harman and Seaton (1966) point up some of the difficulties involved. One may interpret the $\mathrm{H}$ and He II line intensities with the aid of Mihalas' (1965) model atmospheres with $\log g=4 \cdot 0$ and $N(\mathrm{He}) / \mathrm{N}(\mathrm{H})=0 \cdot 15$. We find the following comparison with Seaton's (1966) results:

\section{Central star temperatures (thousands of degrees)}

\begin{tabular}{|c|c|c|c|c|c|c|c|c|c|c|c|}
\hline Object & sec. & LHA & MJS & Object & spec. & LHA & MJS & Object & spec. & LHA & MJS \\
\hline 2149 & $07 \cdot 5$ & $35: 2$ & 49 & 6058 & 09? & 33 & 72 & NC & 07 & 39 & \\
\hline JGC 2392 & 06 & $43: 5$ & 67 & 4593 & 06 & $41: 2$ & & NGC 6853 & 07 & $40: 2$ & 132 \\
\hline NGC 4361 & 06 & 40 & - & NGC 6210 & 06 & $42 \div 2$ & 50 & NGC 6891 & 07 & 37 & 55 \\
\hline 3568 & 05 & 50 & - & NGC 6804 & 09 & $33: 1$ & 72 & NGC 7008 & 07 & 37 & \\
\hline
\end{tabular}

Miss Heap, who is examining this problem in detail, expresses the hope that discrepancies may be eliminated for some objects such as the nuclei of IC 2149 , NGC 6210, and NGC 6891; those of NGC 6853 and 7008 present problems.

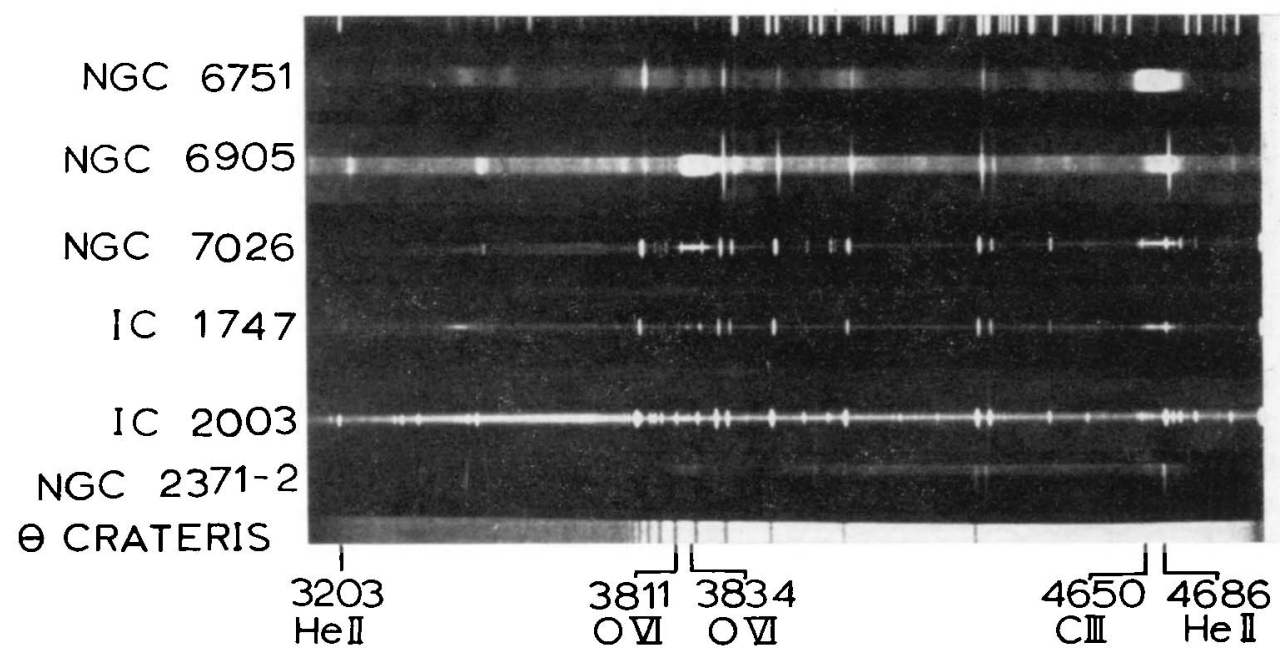

Fig. 7. Comparison of the spectra of several stars of the Wolf-Rayet type. 
If one uses Minkowski's distance scale (quoted by Aller, 1965), O’Dell's (1963) bolometric corrections, central star magnitudes, and temperatures when spectral data are not available, the stars listed in Table 1 are found to yield an HR diagram that shows considerable scatter, neither confirming nor contradicting the sequence proposed by Seaton (1966). Very real uncertainties in the distance scale in addition to those in the temperature and bolometric corrections obliterate any well-defined evolutionary sequences that may exist. Also, if many of these central stars are binaries as seems indeed true for NGC 1514 (Kohoutek, 1966), it would be very difficult to construct a meaningful HR diagram.

O'Dell and others have tried to segregate the various types of stars in the HR diagram and it may be that if lower temperatures and luminosities are accepted for the absorption-line stars, the different types of spectra may correspond to different domains in an HR diagram.

\section{Summary}

Spectra of planetary nuclei obtained with the Lick 120 -inch telescope give additional data for a number of stars heretofore inadequately observed and give more detailed information on a number of the brighter stars. Characteristics of nuclei with WolfRayet-like spectra are shown in detail.

The contradiction between stellar temperatures deduced from Zanstra methods and those appropriate to their spectral classes is found to hold for additional objects. Thus radii and luminosities deduced for these stars must be taken with reserve pending resolution of this difficulty. It is suggested that uncertainties in our present data on distances, temperatures and luminosities, preclude the deduction of precise evolutionary tracks for these stars.

\section{Acknowledgements}

Expenses for necessary travel to Lick Observatory to secure observations were supplied from University of California, Los Angeles and NASA Grant NsG 237-62. Reductions of all observational data were supported by AFOSR Grant 83-65 to UCLA from Air Force Office of Scientific Research. Bruce Bohanan assisted in the reductions of the data for NGC 6543. I am grateful to Merle Walker for permission to reproduce the tracing of the spectrum of the NGC 40 nucleus obtained with the Lallemand cell. Particular gratitude is due to Miss Lindsey Smith for her careful review of the manuscript and for many helpful discussions of problems of Wolf-Rayet stars.

\section{References}

Abell, G.O. (1966)

Aller, L.H. (1943)

Aller, L.H. (1948)

Aller, L. H. (1956)
Astrophys. J., 144, 259.

Astrophys. J., 97, 135.

Astrophys. J., 108, 462.

Gaseous Nebulae, Chapman \& Hall, London. 
Aller, L.H. (1965) Landolt-Bornstein Tables, Group VI, Vol. I in the series: Springer, Berlin, p. 566.

Andrillat, Y., Andrillat, H. (1959) Ann. Astrophys., 22, 104.

Campbell, W.W. (1894) Astronomy and Astrophysics, 13, 461.

Harman, R.J., Seaton, M.J. (1966) Mon. Not. R. astr. Soc., 132, 15.

Kohoutek, L. (1967) Bull. astr. Inst. Csl., 18, 103.

Mihalas, D. (1965) Astrophys. J. Suppl., 9, 321.

O'Dell, C.R. (1963) Astrophys. J., 138, 67.

Minkowski, R., Greenstein, J. (1964) Astrophys. J., 140, 1601.

Oke, J. B. (1954) Astrophys. J., 120, 22.

Paddock, G.F. (1915) Lick Obs. Bull. 9, 30.

Seaton, M.J. (1966) Mon. Not. R. astr. Soc., 132, 113.

Swings, P. (1940) Astrophys. J., 92, 289.

Swings, P., Struve, O. (1940) Proc. nat. Acad. Sci. Am., 26, 454, 548.

Swings, P., Swensson, J. W. (1952) Ann. Astrophys., 15, 290.

Wilson, O.C. (1948) Astrophys. J., 108, 201.

Wilson, O.C., Aller, L.H. (1954) Astrophys. J., 119, 243. 